\title{
Respuesta secretora de PYY1-36 y PYY3-36 en sujetos normales tras la ingesta de una comida mixta
}

\author{
PYY1-36 and PYY3-36 secretory response after a mixed meal in healthy individuals
}

\author{
Manuel Penín ${ }^{\mathrm{a}}$, Miguel Pérez Fontán ${ }^{\mathrm{b}}$, Jesús García Buela ${ }^{\mathrm{d}}$, María Luisa Isidro ${ }^{\mathrm{a}}$, \\ Teresa Martínez ${ }^{\mathrm{a}}$, Elena Outeiriño ${ }^{\mathrm{a}}$, Ana Rodríguez-Carmona ${ }^{\mathrm{b}}$, Susana Sangiao- \\ Alvarellos $^{\mathrm{c}, \mathrm{e}}$, OvidioVidal $^{\mathrm{a}}$, Fernando Cordido ${ }^{\mathrm{a}, \mathrm{c}, \mathrm{e}}$
}

\author{
${ }^{a}$ Servicio de Endocrinología. Hospital Juan Canalejo. A Coruña. España \\ ${ }^{b}$ Servicio de Nefrología. Hospital Juan Canalejo. A Coruña. España \\ ${ }^{c}$ Departmento de Medicina. Universidad de A Coruña. A Coruña. España \\ ${ }^{d}$ Laboratorio de Hormonas. Hospital Juan Canalejo. A Coruña. España \\ ${ }^{e}$ Unidad de Investigación. Hospital Juan Canalejo. A Coruña. España
}

\begin{abstract}
Resumen
Introducción. El péptido YY (PYY) tiene 36 aminoácidos y lo sintetizan fundamentalmente las células L del intestino. El PYY aumenta tras las comidas y alcanza su nadir tras el ayuno. Tras la ingestión se liberan dos formas: $\mathrm{PYY}_{1-36}$ y $\mathrm{PYY}_{3-36}$. Se ha demostrado que el $\mathrm{PYY}_{3-36}$ reduce la ingesta en humanos y roedores. Hay poca información sobre los valores plasmáticos de PYY, especialmente de $\mathrm{PYY}_{3-36}$, en respuesta a la ingestión y su relación con la respuesta de ghrelina.

Objetivos. Estudiar la respuesta secretora de $\mathrm{PYY}_{1-36}$ y $\mathrm{PYY}_{3-36}$ en sujetos normales tras ingerir una comida mixta y su relación con la secreción de ghrelina total y acilada.

Sujetos y métodos. Estudiamos a 8 sujetos sanos, 4 mujeres y 4 varones, con una mediana de edad de 53 (intervalo, 36-59) años. Tras el ayuno nocturno, recibieron en 2 días diferentes y de forma aleatoria: una comida oral mixta estándar, que consistía en $400 \mathrm{ml}$ de Isosource Energy $(159 \mathrm{kcal} / 100 \mathrm{ml})$ o placebo por vía oral (400 ml de agua). Se obtuvieron muestras sanguíneas en los tiempos 0, 30, 45, 60 y 120 min para la determinación de $\mathrm{PYY}_{1-36}, \mathrm{PYY}_{3-36}$, ghrelina total y ghrelina acilada. Las comparaciones se realizaron mediante la prueba de Wilcoxon. Las correlaciones numéricas se analizaron mediante la prueba de correlación de Spearman. Se consideró significativo un valor de $\mathrm{p} \leq 0,05$.

Resultados. Tras ingerir la comida, se produce un máximo de PYY ${ }_{1-36}$ (mediana [intervalo]) de 141,5 (81-198) $\mathrm{pg} / \mathrm{ml}$ y no hay respuesta tras placebo, con un máximo de 92,5 $(46-219) \mathrm{pg} / \mathrm{ml}(\mathrm{p}=0,04)$. Los valores del área bajo la curva $(\mathrm{ABC})$ de $\mathrm{PYY}_{1-36}$ tras la ingesta fueron $14.865(8.032-19.822) \mathrm{pg} / \mathrm{ml} / \mathrm{min}$ y tras placebo, 8.992 (4.455- 21.382) $\mathrm{pg} / \mathrm{ml} / \mathrm{min}(\mathrm{p}=0,06)$. Tras ingerir la comida se produce un máximo de $\mathrm{PYY}_{3-36}$ de 92,5 (59-135) $\mathrm{pg} / \mathrm{ml}$ y no hay respuesta tras placebo, con un máximo de 46,5 (30-66) $\mathrm{pg} / \mathrm{ml}(\mathrm{p}=0,02)$. Los valores del ABC de PYY $_{3-36}$ tras la ingesta fueron 9.086 (6.412-14.970) pg/ml/min y tras placebo, 4.984 (3.142-6.772) pg/ml/min $(\mathrm{p}=0,012)$. El cociente nadir de ghrelina total/máximo de $\mathrm{PYY}_{1-36}$ disminuye de forma marcada tras la ingestión; los valores preprandiales son 7,44 (3,64-14,56) y los posprandiales, 3,55 $(1,64-7,16)(\mathrm{p}=0,03)$, mientras que no se modifica tras placebo. El cociente nadir de ghrelina acilada/máximo de $\mathrm{PYY}_{3-36}$ disminuye de forma marcada tras la ingestión, y los valores preprandiales son 2,03 (0,92-3) y los posprandiales, $0,73(0,26$ $1,27)(\mathrm{p}=0,02)$, mientras que no se modifican tras placebo.

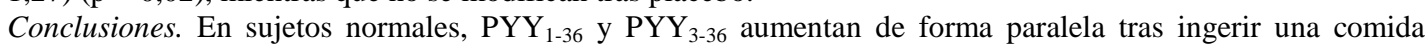
mixta; simultáneamente, los valores de ghrelina total y acilada disminuyen. El cociente entre el nadir de ghrelina acilada y el máximo de $\mathrm{PYY}_{3-36}$ disminuye tras ingerir una comida mixta. Este conjunto de datos indica su posible participación en la regulación aguda del apetito tras la comida.
\end{abstract}




\begin{abstract}
Background. Peptide YY (PYY) is a 36 amino acid peptide synthesized mostly by intestinal L cells. This peptide reaches its nadir during fasting and increases immediately after meals. After food intake, two molecular forms are released, $\mathrm{PYY}_{1-36}$ and $\mathrm{PYY}_{3-36}$. $\mathrm{PYY}_{3-36}$ reduces food intake in both humans and rodents. There is scarce information about plasmatic concentrations of PYY, especially of $\mathrm{PYY}_{3-36}$, after food ingestion, and their relationship to ghrelin.

Objectives. To study $\mathrm{PYY}_{1-36}$ and $\mathrm{PYY}_{3-36}$ secretory response after a mixed meal, and its relationship to total and acylated ghrelin secretion in healthy subjects.

Subjects and method. We studied eight healthy subjects, 4 women and 4 men, with a median age of 53 (range, 36-59) years. After an overnight fast, the subjects received either a mixed standard meal (400 ml Isosource Energy® [159 kcal/100 ml]) or placebo (400 ml of water) orally in random order on two different days. Blood samples were obtained at 0, 30, 45, 60 and 120 min for measurement of $\mathrm{PYY}_{1-36}, \mathrm{PYY}_{3-36}$, total ghrelin and acylated ghrelin. Comparisons were made by Wilcoxon's test. Numerical correlations were performed using Spearman's test. P-values $\leq 0.05$ were considered significant.

Results. After a mixed meal, PYY 1-36 reached a peak of (median [range]) 141.5 (81-198) pg/ml. There was no response to placebo, with a peak of $92.5(46-219) \mathrm{pg} / \mathrm{ml}(\mathrm{p}=0.04)$. The area under the curve (AUC) of PYY $\mathrm{PY}_{1-36}$ levels after a mixed meal were $14,865(8,032-19,822) \mathrm{pg} / \mathrm{ml} / \mathrm{min}$ and after placebo were $8,992(4,455-21,382)$ $\mathrm{pg} / \mathrm{ml} / \mathrm{min}(\mathrm{p}=0.06)$. After ingestion of a mixed meal, PYY ${ }_{3-36}$ reached a peak of $92.5(59-135) \mathrm{pg} / \mathrm{ml}$, with no response to placebo $(46.5[30-66] \mathrm{pg} / \mathrm{ml})(\mathrm{p}=0.02)$. The AUC of PYY $3-36$ levels after a mixed meal were 9,086 $(6,412-14,970) \mathrm{pg} / \mathrm{ml} / \mathrm{min}$, and after placebo were 4,984 $(3,142-6,772) \mathrm{pg} / \mathrm{ml} / \mathrm{min}(\mathrm{p}=0.012)$. The quotient between nadir total ghrelin/peak PYY 1-36 $_{\text {was }}$ markedly diminished after food ingestion, with preprandial values of $7.44(3.64-14.56)$ and postprandial values of $3.55(1.64-7.16)(\mathrm{p}=0.03)$. The former quotient was unmodified by placebo. The quotient between nadir acylated ghrelin/peak $\mathrm{PYY}_{3-36}$ was markedly diminished after ingestion of a mixed meal, with preprandial values of $2.03(0.92-3)$ and postprandial values of $0.73(0.26-1.27)(\mathrm{p}=0.02)$. This quotient was unmodified by placebo.

Conclusions. In healthy subjects, blood levels of both $\mathrm{PYY}_{1-36}$ and $\mathrm{PYY}_{3-36}$ increase after ingestion of a mixed meal. Simultaneously, total and acylated ghrelin levels diminish. The quotient between nadir acylated ghrelin/peak PYY ${ }_{3-36}$ diminishes after a mixed meal. All these data suggest the possible contribution of these peptides to appetite regulation after ingestion.
\end{abstract}

\title{
Palabras clave
}

Secreción; $\mathrm{PYY}_{1-36 ;} \mathrm{PYY}_{3-36 ;}$ Ingesta

Key words

Secretion; $\mathrm{PYY}_{1-36 ;} \mathrm{PYY}_{3-36}$; Ingestion

\section{INTRODUCCIÓN}

El péptido al que actualmente se concede mayor importancia como regulador fisiológico precoz de la ingesta probablemente sea el péptido YY (PYY). PYY tiene 36 aminoácidos y lo sintetizan fundamentalmente las células L del intestino. El PYY se encuentra por todo el intestino, pero sus mayores concentraciones se producen en los segmentos distales ${ }^{1}$. Tras comer se liberan dos formas ${ }^{1-3}$ : $\mathrm{PYY}_{1-36}$ y PYY $_{3-36}$. Batterham et al ${ }^{4,5}$ han demostrado que PYY ${ }_{3-36}$ reduce la ingesta en humanos y roedores, aunque una publicación reciente ha encontrado que el PYY es incapaz de inhibir la ingesta en roedores ${ }^{6}$. Más recientemente se ha visto que para la demostración del efecto anorexigénico del PYY se require la habituación de los animales a la manipulación e inyección ${ }^{4,7,8}$. El PYY, por lo tanto, puede ser un factor fundamental en el control de la saciedad posprandial ${ }^{9,10}$. La concentración plasmática posprandial de PYY es proporcional a la cantidad de comida ${ }^{1}$, y en algún estudio se alcanza el máximo plasmático sobre unas $2 \mathrm{~h}$ después de la comida ${ }^{4,5}$. Se han descrito elevadas cantidades de PYY en ayunas en diversas enfermedades gastrointestinales asociadas con disminución del apetito ${ }^{11,12}$. La concentración de PYY en ayunas más baja se ha encontrado en los pacientes obesos $^{5}$. Las diferencias en la concentración de PYY en ayunas son controvertidas ${ }^{13}$; sin embargo, parece que la duración del ayuno antes de un estudio afecta de forma muy importante a las concentraciones plasmáticas de $\mathrm{PYY}^{14}$. Aunque se ha demostrado una disminución de la respuesta posprandial del PYY en pacientes obesos ${ }^{5,13,15}$, en pacientes obesos se asocia con disminución de la saciedad ${ }^{16}$. 
Hay poca información publicada sobre la concentración plasmática de PYY en respuesta a cambios en el balance energético. El PYY aumenta tras las comidas y alcanza su nadir tras el ayuno. Según transcurre el día, la concentración de PYY es menor en ayunas y aumenta tras el desayuno, la comida y la cena ${ }^{17}$. Tras ingerir una comida de prueba, la concentración aumenta a los 15 min de forma proporcional a la cantidad de calorías ingeridas, alcanza el máximo sobre los $90 \mathrm{~min}$ y permanece elevada hasta $6 \mathrm{~h}$ después ${ }^{1}$. De las dos formas del péptido que se secretan tras la ingesta $\mathrm{PYY}_{1-36}$ y $\mathrm{PYY}_{3-36^{-}}$, la segunda tiene una acción anorexígena fisiológica. Igualmente existen pocos estudios sobre la secreción de $\mathrm{PYY}_{3-36}$, la forma fisiológicamente activa en sujetos normales tras la ingestión y se han realizado en un escaso número de pacientes ${ }^{16,18}$. No se ha establecido tras la ingestión la relación de las dos formas circulantes del PYY con la ghrelina acilada.

La ghrelina es un péptido de 28 aminoácidos sintetizado por el estómago y presenta un grupo éster N-octanoil en la serina $3^{19-23}$. La actividad orexigénica de la ghrelina se descubrió por primera vez en un modelo animal ${ }^{24,25}$. La ghrelina actúa, fundamentalmente, uniéndose al receptor de los secretagogos de GH 1a (GHS-R1a) en el núcleo arcuato. Este receptor participa en la regulación de la secreción de $\mathrm{GH}$, la regulación de la ingesta y la adiposidad ${ }^{26}$. La infusión de ghrelina aumenta el hambre a corto plazo en el humano ${ }^{27}$. Existen diferentes formas moleculares de ghrelina circulantes. La forma activa de ghrelina, desde el punto de vista endocrino y metabólico, necesita un grupo $\mathrm{N}$ octanoil en la serina $3^{28-30}$, pues la forma no acilada no se une al receptor GHS-R $1 a^{19,30,31}$.

El objetivo de este trabajo es estudiar la respuesta secretora de $\mathrm{PYY}_{1-36}$ y $\mathrm{PYY}_{3-36}$ en sujetos normales tras ingerir una comida mixta y su relación con la secreción de ghrelina total y acilada.

\section{SUJETOS Y MÉTODO}

Hemos estudiado en sujetos sanos los valores basales en ayunas y la respuesta aguda de PYY $\mathrm{PY}_{1-36} \mathrm{y}$ $\mathrm{PYY}_{3-36}$ tras ingerir una comida mixta o placebo, mediante un diseño aleatorio. Por lo tanto, en cada sujeto se realizaron dos pruebas (comida mixta o placebo) de forma aleatoria, y cada sujeto sirvió como su propio control. Las pruebas comenzaban a las nueve de la mañana tras el ayuno nocturno, con los sujetos en decúbito supino. Se insertaba un catéter en el antebrazo para las extracciones sanguíneas. El protocolo de estudio fue aprobado por el comité ético de nuestro centro y se obtuvo consentimiento informado de todos los sujetos.

Estudiamos a 8 sujetos sanos, 4 mujeres y 4 varones, con una mediana (intervalo) de edad de 53 (36-59) años. La mediana del índice de masa corporal (IMC) fue 25,1 (20,1-29,3). Ninguno de los sujetos presentaba diabetes mellitus u otros problemas médicos ni tomaba fármacos. Este estudio se ha realizado en sujetos de mediana edad, entre 35 y 65 años, porque la mayoría de los estudios se han realizado en sujetos más jóvenes. Todos los sujetos presentaban valores en ayunas dentro del rango de la normalidad en glucemia, 97 (85-103) mg/dl; colesterol, 218 (179-236) mg/dl; triglicéridos, 134 (103-148) $\mathrm{mg} / \mathrm{dl}$; insulinemia, 9,6 (3,7-15,1) $\mu \mathrm{U} / \mathrm{ml}$, y valor HOMA (Homeostasis Model Assessment), 2,4 (0,6-3,9).

Los sujetos fueron aconsejados para que mantuvieran una dieta normocalórica, con el objeto de mantener el peso estable en las semanas previas a los estudios.

Tras el ayuno nocturno, a las 9.00 los sujetos permanecían en reposo en decúbito y se les colocaba una vía venosa periférica; 30 min más tarde recibían en 2 días diferentes:

- Una comida oral mixta estándar, que consistía en $400 \mathrm{ml}$ de Isosource Energy® (Novartis, Zúrich, Suiza), con 5,7 g de proteína, 6,2 g de lípidos y 20 g de hidratos de carbono cada 100 $\mathrm{ml}(159 \mathrm{kcal} / 100 \mathrm{ml})$.

- Placebo: $400 \mathrm{ml}$ de agua por vía oral.

Se obtuvieron muestras sanguíneas en los tiempos 0, 30, 45, 60 y 120 min para la determinación de $\mathrm{PYY}_{1-36}, \mathrm{PYY}_{3-36}$, ghrelina total y ghrelina acilada. Los datos en relación con la respuesta de ghrelina total, ghrelina acilada, leptina, insulina y GH durante la misma prueba han sido publicados previamente $^{32}$. 
Todas las muestras sanguíneas fueron específicamente recogidas en tubos en frío con aprotinina y EDTA-Na e inmediatamente centrifugadas a $4{ }^{\circ} \mathrm{C}$, separadas en alícuotas y congeladas a $-80^{\circ} \mathrm{C}$.

Las determinaciones hormonales se realizaron mediante los siguientes métodos comerciales: PYY $_{1-36}$ con RIA (Linco, St. Charles, Misuri, Estados Unidos), con coeficientes de variación intraanalítica e interanalítica del 2,9 y el 5,5\% respectivamente y un límite de detección de $10 \mathrm{pg} / \mathrm{ml}$; $\mathrm{PYY}_{3-36}$ con RIA (Linco), con coeficientes de variación intraanalítica e interanalítica del 6,4 y el 7\% respectivamente y un límite de detección de $20 \mathrm{pg} / \mathrm{ml}$; ghrelina total con RIA (Phoenix Pharmaceuticals, Belmont, California, Estados Unidos), con coeficientes de variación intraanalítica e interanalítica del 5,3 y el 13,6\% respectivamente; ghrelina acilada con RIA (Linco), con coeficientes de variación intraanalítica e interanalítica del 6,5 y el 9,6\% respectivamente y un límite de detección de $10 \mathrm{pg} / \mathrm{ml}$.

La respuesta temporal de los valores de $\mathrm{PYY}_{1-36}$ y $\mathrm{PYY}_{3-36}$ tras las diferentes pruebas se analizaron en valores absolutos. El área bajo la curva $(\mathrm{ABC})$ se determinó por el método trapezoidal.

Todas las comparaciones se realizaron mediante pruebas no paramétricas. El grado de concordancia entre las estimaciones basales se analizó mediante el coeficiente de correlación de Spearman y la prueba de la t de Student para datos apareados (sesgo sistemático). Las comparaciones se realizaron mediante la prueba de Wilcoxon. Las correlaciones numéricas se analizaron mediante la prueba de correlación de Spearman. Se consideró significativo un valor de $\mathrm{p} \leq 0,05$. Los valores numéricos se presentan en forma de mediana (intervalo). Los valores gráficos se presentan en forma de media \pm error estándar de la media (EEM). El análisis estadístico se realizó mediante el programa estadístico SPSS 14.0.

\section{RESULTADOS}

Los valores basales preprandiales y posprandiales tras las dos pruebas se presentan en la tabla 1.

Tabla 1. Valores preprandriales y posprandiales y relaciones de $\mathrm{PYY}_{1-36}, \mathrm{PYY}_{3-36}$, ghrelina total y ghrelina acilada

\begin{tabular}{|c|c|c|c|}
\hline & Preprandial & Posprandial & $\mathrm{p}$ \\
\hline \multicolumn{4}{|l|}{$\mathrm{PYY}_{1-36}(\mathrm{pg} / \mathrm{ml})$} \\
\hline Comida mixta & $100(61-179)$ & $141,5(81-198)$ & $<0,05$ \\
\hline Placebo & $73(52-164)$ & $92,5(46-219)$ & NS \\
\hline \multicolumn{4}{|l|}{$\mathrm{PYY}_{3-36}(\mathrm{pg} / \mathrm{ml})$} \\
\hline Comida mixta & $70(51-89)$ & $92,5(59-135)$ & $<0,05$ \\
\hline Placebo & $56(37-71)$ & $46,5(30-66)$ & NS \\
\hline \multicolumn{4}{|c|}{ Ghrelina total (pg/ml) } \\
\hline Comida mixta & $731(399-1016)$ & $530(352-587)$ & $<0,05$ \\
\hline Placebo & $669(411-1023)$ & $693(432-1109)$ & NS \\
\hline \multicolumn{4}{|c|}{ Ghrelina acilada (pg/ml) } \\
\hline Comida mixta & $140(43-193)$ & $68(22-81)$ & $<0,05$ \\
\hline Placebo & $112(39-227)$ & $124(43-231)$ & NS \\
\hline \multicolumn{4}{|c|}{ Cociente ghrelina total/PYY ${ }_{1-36}$} \\
\hline Comida mixta & $7,44(3,64-14,56)$ & $3,55(1,64-7,16)$ & $<0,05$ \\
\hline Placebo & $9,84(2,51-20,95)$ & $7,49(2,78-24,21)$ & NS \\
\hline \multicolumn{4}{|c|}{ Cociente ghrelina acilada/PYY } \\
\hline Comida mixta & $2,03(0,92-3,00)$ & $0,73(0,26-1,27)$ & $<0,05$ \\
\hline Placebo & $2,14(0,74-3,85)$ & $2,67(0,75-5,7)$ & NS \\
\hline
\end{tabular}

Las comparaciones se realizan con la prueba de Wilcoxon entre valores preprandiales y posprandiales. 
Los valores de PYY ${ }_{1-36}$ y $\mathrm{PYY}_{3-36}$ presentaban una buena correlación. Sin embargo, de forma un tanto sorprendente, los valores basales de $\mathrm{PYY}_{3-36}$ fueron menores durante la prueba de placebo que tras la ingestión (tabla 1). La correlación entre los valores plasmáticos basales de $\mathrm{PYY}_{1-36}$ y $\mathrm{PYY}_{3-36}$ en la prueba de ingesta oral fue $r=0,38(p=0,31)$ y en la prueba de placebo, $r=0,78(p=0,03)$.

No encontramos correlaciones claras entre los valores basales de $\mathrm{PYY}_{1-36}$ y $\mathrm{PYY}_{3-36}$, por un lado, y los valores de ghrelina total y acilada, por el otro. No encontramos correlación aparente entre los valores basales de $\mathrm{PYY}_{1-36}$ y $_{\mathrm{PY}} \mathrm{Y}_{3-36}$, por un lado, y las variables demográficas, por otro (edad, sexo, IMC), posiblemente en parte por el pequeño número de sujetos estudiados para este objetivo.

Los cocientes entre los valores basales de ghrelina y PYY se presentan en la tabla 1. Estos cocientes, que se podría interpretar como una puntuación orexigénica, disminuyen marcadamente tras la ingestión.

Los valores plasmáticos de PYY $\mathrm{PY}_{1-36}$ tras una comida mixta o placebo se muestran en la figura 1. La ingestión de una comida mixta causa un claro incremento en los valores de PYY ${ }_{1-36}$.

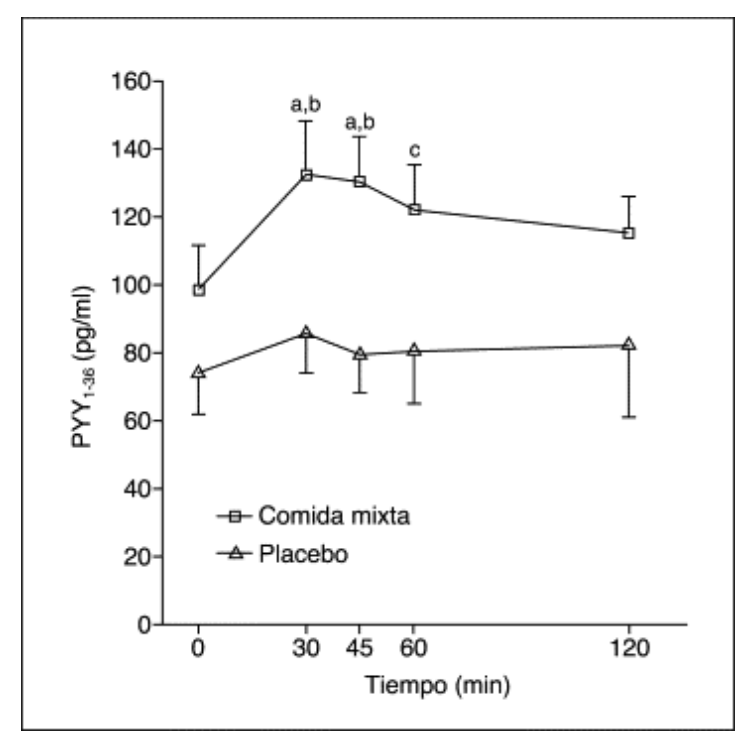

Fig 1. Valores plasmáticos de $P Y_{\mathrm{I}-36}$ en sujetos sanos tras placebo o una comida mixta. Los valores se presentan en forma de media \pm error estándar de la media.

Tras la comida se produce un máximo valor de PYY $\mathrm{P}_{1-36}-141,5$ (81-198) pg/ml-, pero no hay respuesta tras placebo, con un máximo de 92,5 (46-219) pg/ml (p = 0,04). Los valores del ABC de $\mathrm{PYY}_{136}$ tras la ingesta fueron $14.865(8.032-19.822) \mathrm{pg} / \mathrm{ml} / \mathrm{min}$ y tras placebo, $8.992(4.455-21.382)$ $\mathrm{pg} / \mathrm{ml} / \mathrm{min}(\mathrm{p}=0,06)$.

Los valores plasmáticos de $\mathrm{PYY}_{3-36}$ tras una comida mixta o placebo se muestran en la figura 2. La ingestión de una comida mixta causa un claro incremento en los valores de $\mathrm{PYY}_{3-36}$. 


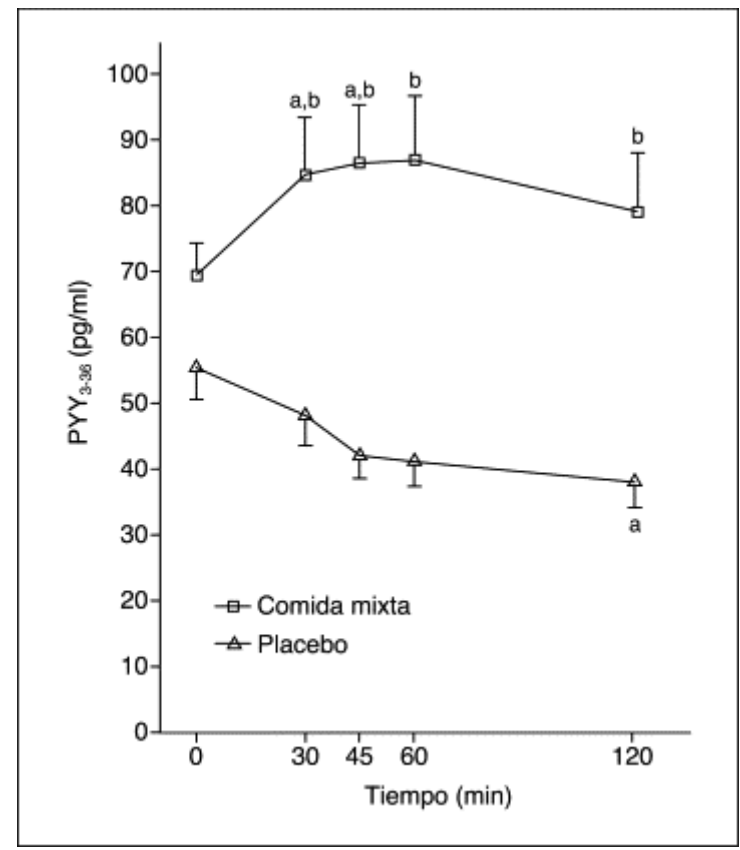

Fig. 2. Valores plasmáticos de $\mathrm{PYY}_{3-36}$ en sujetos sanos tras placebo o una comida mixta. Los valores se presentan en forma de media \pm error estándar de la media. ${ }^{\mathrm{a}} \mathrm{p}<0,05$ frente a basal. ${ }^{\mathrm{b}}$ Placebo frente a comida, $\mathrm{p}<0,02$ en todos los puntos tras basal.

Tras ingerir la comida se produce un máximo de PYY $\mathrm{P}_{3-36}$ de 92,5 (59-135) pg/ml, pero no hay respuesta tras placebo, con un máximo de 46,5 (30-66) pg/ml $(\mathrm{p}=0,02)$. Los valores del ABC de $\mathrm{PYY}_{3-36}$ tras la ingesta fueron $9.086(6.412-14.970) \mathrm{pg} / \mathrm{ml} / \mathrm{min}$ y tras placebo, $4.984(3.142-6.772)$ $\mathrm{pg} / \mathrm{ml} / \mathrm{min}(\mathrm{p}=0,012)$.

Estudiamos la relación entre el $\mathrm{ABC}$ de $\mathrm{PYY}_{1-36}$ por un lado y el $\mathrm{ABC}$ de los valores de glucemia, insulina, ghrelina total, ghrelina acilada, GH o leptina, por otro, sin encontrar correlaciones estadísticamente significativas.

Estudiamos la relación entre el ABC de $\mathrm{PYY}_{3-36}$ por un lado y el ABC de los valores de glucemia, insulina, ghrelina total, ghrelina acilada, GH o leptina, por otro, sin encontrar correlaciones estadísticamente significativas.

No encontramos relación entre los valores de $\mathrm{PYY}_{1-36}$ o PYY $\mathrm{PY}_{36}$ tras la comida y la respuesta de insulina, glucosa, ghrelina total, ghrelina acilada, GH o leptina, lo que indica que ninguno de los referidos factores participa en la regulación del PYY.

El cociente nadir de ghrelina total/máximo de $\mathrm{PYY}_{1-36}$ disminuye de forma marcada tras la comida, y los valores preprandiales son $7,44(3,64-14,56)$ y los posprandiales, $3,55(1,64-7,16)(\mathrm{p}=$ $0,03)$, pero no se modifican tras placebo (tabla 1 ).

El cociente nadir de ghrelina acilada/máximo de $\mathrm{PYY}_{3-36}$ disminuye de forma marcada tras la comida, y los valores preprandiales son $2,03(0,92-3)$ y los posprandiales, $0,73(0,26-1,27)(p=0,02)$, pero no se modifican tras placebo (tabla 1$)$. 


\section{DISCUSIÓN}

En nuestro estudio, las concentraciones de $\mathrm{PYY}_{1-36}$ y $\mathrm{PYY}_{3-36}$ en sujetos sanos aumentan de forma paralela tras la ingestión de una comida mixta, y simultáneamente las de ghrelina total y acilada disminuyen. El cociente entre el nadir de ghrelina acilada y el máximo de $\mathrm{PYY}_{3-36}$ disminuye tras una comida mixta.

Es objeto de creciente interés el papel de los péptidos gastrointestinales como reguladores primordiales de la ingesta ${ }^{33-36}$. El PYY pertenece a la familia del polipéptido pancreático (PP) y el neuropéptido Y (NPY). El ARNm del PYY ha sido identificado en el tracto gastrointestinal y el páncreas, y al igual que otras hormonas gastrointestinales, se ha identificado en neuronas características del tronco cerebral $^{37}$. El PYY es metabolizado por la enzima dipeptidilpeptidasa IV

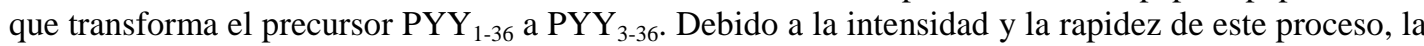
forma primordial circulante en el plasma humano tras la comida es $\mathrm{PYY}_{3-36}$, que posee una importante actividad biológica ${ }^{3}$.

La demostración de la acción anorexígena del $\mathrm{PYY}_{336}$ ha resultado sorprendente, pues la administración directa en el sistema nervioso central de $\mathrm{PYY}_{1-36}$ o $\mathrm{PYY}_{336}$ aumenta la ingesta ${ }^{38}$. Para explicar esta paradoja se ha formulado un modelo basado en los subtipos de receptores $\mathrm{Y}$ y su disponibilidad ${ }^{4}$. El $\mathrm{PYY}_{1-36}$ activa todos los receptores $\mathrm{Y}$, y sus efectos orexigénicos están mediados por su interacción con los receptores $\mathrm{Y} 1 \mathrm{R}$ e $\mathrm{Y} 5 \mathrm{R}$, que se expresan en el núcleo paraventricular hipotalámico y se cree que median el efecto orexigénico del $\mathrm{NPY}^{39}$. El PYY $\mathrm{PY}_{36}$ activa selectivamente Y2R e Y5R, y la administración intracerebroventricular de este péptido puede aumentar la ingesta a través de Y5R. Sin embargo, se cree que el PYY ${ }_{3-36}$ circulante accede selectivamente al Y2R en el núcleo arcuato del hipotálamo, que se encuentra fuera de la barrera hematoencefálica. En el hipotálamo, el Y2R es un receptor presináptico autoinhibitorio de las neuronas orexigénicas que expresan NPY y la proteína relacionada con agouti (AGRP). Por lo tanto, este modelo propone que el $\mathrm{PYY}_{3-36}$ reduce la ingesta inhibiendo las neuronas NPY/AGRP a través de la activación del Y2R, al mismo tiempo que disminuye la represión de las neuronas anorexígenas adyacentes productoras de melanocortina, que se encuentran inhibidas por las neuronas NPY/AGRP ${ }^{4}$. Coherente con este modelo, el efecto de $\mathrm{PYY}_{3-36}$ estimulador de la ingesta se anula con el bloqueo farmacológico o genético del $\mathrm{Y}_{2} \mathrm{R}^{4,40}$. La inyección en el núcleo arcuato de $\mathrm{PYY}_{3-36}$ inhibe la ingesta, mientras que la administración intracerebroventricular tiene el efecto contrario ${ }^{4}$. A pesar de estos hallazgos que confirmarían un mecanismo de acción hipotalámico de la administración periférica de $\mathrm{PYY}_{3-36}$, se ha propuesto también una mediación vagal ${ }^{41}$. Los efectos anorexígenos de $\mathrm{PYY}_{3-36}$ no han sido demostrados por todos los grupos ${ }^{6}$, aunque sí por la mayoría de ellos ${ }^{7,40,42}$. Recientes estudios en humanos con PYY $_{3-36}$ administrado por vía intranasal no han podido arrojar resultados concluyentes, posiblemente en parte por mala tolerancia a las dosis anorexígenas clínicamente eficaces ${ }^{43}$.

En el presente estudio, tras la ingestión de una comida mixta de $600 \mathrm{kcal}$, los valores de PYY 1-36 alcanzan un máximo a los $30 \mathrm{~min}$. Los de $\mathrm{PYY}_{3-36}$ lo alcanzan a los $45 \mathrm{~min}$, y permanecen elevados hasta el final de la prueba, a los $120 \mathrm{~min}$. En el estudio de le Roux et a ${ }^{16}$, los valores máximos de PYY se alcanzan a los 90 min, aunque las determinaciones se realizan cada 30 min y la ingestión calórica fue variable, entre 250 y $3.000 \mathrm{kcal}$. El PYY es sintetizado y liberado por células endocrinas especializadas (células L) que se encuentran fundamentalmente en el tracto gastrointestinal distal. En nuestro estudio, en respuesta a la ingestión de nutrientes, los valores plasmáticos tanto de PYY 1-36 como de $\mathrm{PYY}_{3-36}$ responden de forma precoz, y después permanecen elevados los de $\mathrm{PYY}_{3-36}$. El incremento inicial se produce antes de que los nutrientes alcancen las células L, lo que implica mecanismos neurales, posiblemente vagales y endocrinos. La liberación más sostenida posiblemente se deba al efecto directo del contenido intraluminal gástrico en las células $\mathrm{L}^{44}$. Otros autores ${ }^{16,18,45}$ han encontrado resultados similares, aunque con un diseño experimental diferente. El estudio actual aporta, fundamentalmente, información adicional sobre el posible papel de la forma activa circulante del PYY, PYY ${ }_{3-36}$, como factor de la regulación aguda de la ingesta.

Los valores de PYY $_{3-36}$ estaban moderada aunque significativamente elevados antes de la ingestión de la comida mixta, comparado con los valores antes de la ingestión del placebo. Este hallazgo podría deberse a un aumento de la actividad vagal, activado por la anticipación de la comida; algo similar se ha encontrado para la concentración de insulina tras el ayuno ${ }^{46}$. Igualmente se ha demostrado que el sham-feeding, en el que se visualiza, se huele, se mastica y se saborea, pero no se deglute la comida, es capaz de disminuir la cantidad total de ghrelina ${ }^{47}$. 
Es igualmente importante el papel de la ghrelina en la regulación de la ingesta en humanos, pues la infusión aguda de ghrelina aumenta el hambre a corto plazo ${ }^{27}$. Los valores plasmáticos de ghrelina se correlacionan de forma negativa con el índice de masa corporal, la masa grasa, la leptina, la insulina y la glucosa ${ }^{28,48-50}$. La concentración de ghrelina circulante aumenta antes de las comidas y disminuye tras ellas ${ }^{51}$. La distensión gástrica con agua no disminuye la cantidad de ghrelina ${ }^{52,53}$; sin embargo, la ingestión de fibra no nutritiva reduce la ghrelina ${ }^{54}$. La ghrelina acilada disminuye igualmente tras la ingestión de una comida mixta ${ }^{55-57}$. Nuestro grupo estudia desde hace años el papel de la ghrelina y otros péptidos en situaciones con alteración del balance nutricional ${ }^{22,58-61}$. Nosotros hemos encontrado que la ghrelina total y la acilada disminuyen tras la ingestión de una comida mixta en sujetos normales y que esta respuesta se correlaciona inversamente con la secreción de insulina ${ }^{62}$. El PYY se secreta de forma inversa a la forma activa de la ghrelina en el apetito, la ghrelina acilada. Estos datos indican que ambos péptidos podrían actuar de forma coordinada para regular de forma aguda la ingesta. No encontramos relación entre los valores de $\mathrm{PYY}_{1-36}$ o los de $\mathrm{PYY}_{3-36}$ tras la comida y la respuesta de insulina, glucosa, ghrelina total, ghrelina acilada, GH o leptina, lo que indica que ninguno de esos factores participa en la regulación del PYY. Estos datos contrastan en parte con los de Giménez-Palop et al ${ }^{63}$, que encuentran una correlación inversa entre el incremento de PYY y la disminución de ghrelina a los 60 y los 120 min tras la ingestión, aunque las condiciones del estudio no son superponibles. Los valores de PYY tras la sobrecarga oral de glucosa se han encontrado más elevados en la mujer que en el varón. Por otra parte, en ese mismo estudio no se encontró correlación entre los valores de PYY en ayunas o tras la ingestión y el $\mathrm{IMC}^{64}$.

En resumen, en sujetos sanos, las concentraciones de PYY 1-36 $_{\text {y }} \mathrm{PYY}_{3-36}$ aumentan de forma paralela tras una comida mixta y simultáneamente las de ghrelina total y las de acilada disminuyen. El cociente entre el nadir de ghrelina acilada y el máximo de PYY $\mathrm{PY}_{36}$ disminuye tras una comida mixta. Este conjunto de datos indica su posible participación en la regulación aguda del apetito tras la ingestión.

\section{Bibliografía}

1. T.E. Adrian, G.L. Ferri, A.J. Bacarese-Hamilton, H.S. Fuessl, J.M. Polak, S.R. Bloom. Human distribution and release of a putative new gut hormone, peptide YY. Gastroenterology, 89 (1985), pp. 1070-1077

2. G.A. Eberlein, V.E. Eysselein, M. Schaeffer, P. Layer, D. Grandt, H. Goebell, et al. A new molecular form of PYY: structural characterization of human PYY(3-36) and PYY(1-36). Peptides, 10 (1989), pp. 797-803

3. D. Grandt, M. Schimiczek, C. Beglinger, P. Layer, H. Goebell, V.E. Eysselein, et al. Two molecular forms of peptide YY (PYY) are abundant in human blood characterization of a radioimmunoassay recognizing PYY 1-36 and PYY 3-36. Regul Pept, 51 (1994), pp. 151-159

4. R.L. Batterham, M.A. Cowley, C.J. Small, H. Herzog, M.A. Cohen, C.L. Dakin, et al. Gut hormone PYY (336) physiologically inhibits food intake. Nature, 418 (2002), pp. 650-654

5. R.L. Batterham, M.A. Cohen, S.M. Ellis, C.W. Le Roux, D.J. Withers, G.S. Frost, et al. Inhibition of food intake in obese subjects by peptide YY3-36. N Engl J Med, 349 (2003), pp. 941-948

6. M. Tschop, T.R. Castaneda, H.G. Joost, C. Thone-Reineke, S. Ortmann, S. Klaus, et al. Physiology: does gut

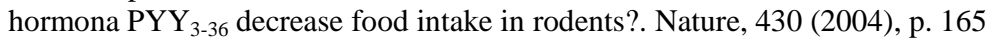

7. I.G. Halatchev, K.L. Ellacott, W. Fan, R.D. Cone. Peptide YY3-36 inhibits food intake in mice through a melanocortin-4 receptorindependent mechanism. Endocrinology, 145 (2004), pp. 2585-2590

8. P.K. Chelikani, A.C. Haver, R.D. Reidelberger. Intravenous infusion of peptide YY(3-36) potently inhibits food intake in rats. Endocrinology, 146 (2005), pp. 879-888

9. M.W. Schwartz, G.J. Morton. Obesity: keeping hunger at bay. Nature, 418 (2002), pp. 595-597

10. C.B. Saper, T.C. Chou, J.K. Elmquist. The need to feed: homeostatic and hedonic control of eating. Neuron, 36 (2002), pp. 199-211

11. T.E. Adrian, A.P. Savage, H.S. Fuessl, K. Wolfe, H.S. Besterman, S.R. Bloom. Release of peptide YY (PYY) after resection of small bowel, colon, or pancreas in man. Surgery, 101 (1987), pp. 715-719

12. T.E. Adrian, A.P. Savage, A.J. Bacarese-Hamilton, K. Wolfe, H.S. Besterman, S.R. Bloom. Peptide YY abnormalities in gastrointestinal diseases. Gastroenterology, 90 (1986), pp. 379-384

13. S. Stock, P. Leichner, A.C. Wong, M.A. Ghatei, T.J. Kieffer, S.R. Bloom, et al. Ghrelin, peptide YY, glucose-dependent insulinotropic polypeptide, and hunger responses to a mixed meal in anorexic, obese, and control female adolescents. J Clin Endocrinol Metab, 90 (2005), pp. 2161-2168

14. S.A. Tovar, L.M. Seoane, J.E. Caminos, R. Nogueiras, F.F. Casanueva, C. Dieguez. Regulation of peptide YY levels by age, hormonal, and nutritional status. Obes Res, 12 (2004), pp. 1944-1950

15. J. Korner, M. Bessler, L.J. Cirilo, I.M. Conwell, A. Daud, N.L. Restuccia, et al. Effects of Roux-en-Y gastric bypass surgery on fasting and postprandial concentrations of plasma ghrelin, peptide YY, and insulin. J Clin Endocrinol Metab, 90 (2005), pp. 359-365 
16. C.W. Le Roux, R.L. Batterham, S.JB. Aylwin, M. Patterson, C.M. Borg, K.J. Wyne, et al. Attenuated peptide YY release in obese subjects is associated with reduced satiety. Endocrinology, 147 (2006), pp. 3-8

17. E.E. Soffer, T.E. Adrian, J. Launspach, B. Zimmerman. Meal-induced secretion of gastrointestinal regulatory peptides is not affected by sleep. Neurogastroenterol Motil, 9 (1997), pp. 7-12

18. P.T. Pfluger, T.R. Kampe, T.R. Castaneda, T. Vahl, D.A. D'Alessio, T. Kruthaupt, et al. Effect of human body weight changes on circulating levels of peptide YY and peptide YY3-36. J Clin Endocrinol Metab, 92 (2007), pp. 583-588

19. M. Kojima, H. Hosoda, Y. Data, M. Nakazato, H. Matsuo, K. Kangawa. Ghrelin is a growth hormonereleasing acylated peptide from stomach. Science, 402 (1999), pp. 656-658

20. A.D. Howard, S.D. Feighner, D.F. Cully, J.P. Arena, P.A. Liberator, C.I. Rosenblum, et al. A receptor in pituitary and hypothalamus that functions in growth hormone release. Science, 273 (1996), pp. 974-976

21. F. Cordido, A. Peñalva, C. Dieguez, F. Casanueva. Massive growth hormone discharge in obese subjects after the combined administration of growth hormone releasing hormone and GHRP-6. Evidence for a marked somatotroph secretory capability in obesity. J Clin Endocrinol Metab, 76 (1993), pp. 819-823

22. P. Alvarez, L. Isidro, J. Garcia-Buela, J. Leal-Cerro, F. Broglio, F. Tassone, et al. Marked GH secretion after ghrelin administration alone or combined with growth hormone releasing hormone (GHRH) in obese patients. Clin Endocrinol, 61 (2004), pp. 250-255

23. A.J. Van der Lely, M. Tschop, M.L. Heiman, E. Ghigo. Biological, physiological, pathophysiological, and pharmacological aspects of ghrelin. Endocr Rev, 25 (2004), pp. 426-457

24. M. Tschop, D.L. Smiley, M.L. Heiman. Ghrelin induces adiposity in rodents. Nature, 407 (2000), pp. $908-$ 909

25. M. Nakazato, N. Murakami, Y. Date, M. Kojima, H. Matsuo, K. Kangawa, et al .A role for ghrelin in the central regulation of feeding. Nature, 409 (2001), pp. 194-198

26. Y. Shuto, T. Shibasaki, A. Otagiri, H. Kuriyama, H. Ohata, H. Tamura, et al. Hypothalamic growth hormone secretagogue receptor regulates growth hormone secretion, feeding and adiposity. J Clin Invest, 109 (2002), pp. $1429-1436$

27. A.M. Wren, L.J. Seal, M.A. Cohen, A.E. Brynes, G.S. Frost, K.G. Murphy, et al. Ghrelin enhances appetite and increases food intake in humans. J Clin Endocrinol Metab, 86 (2001), pp. 5992-5998

28. G. Muccioli, M. Tschop, M. Papotti, R. Deghenghi, M. Heiman, E. Ghigo Neuroendocrine and peripheral activities of ghrelin: implications in metabolism and obesity. Eur J Pharmacol, 440 (2002), pp. 235-254

29. C.Y. Bowers. Unnatural growth hormone releasing peptide begets natural ghrelin. J Clin Endocrinol Metab, 86 (2001), pp. 1464-1469

30. F. Broglio, A. Benso, C. Gottero, F. Prodam, C. Gauna, L. Filtri, et al. Non-acylated ghrelin does not possess the pituitaric and pancreatic endocrine activity of acylated ghrelin in humans. J Endocrinol Invest, 26 (2003), pp. $192-196$

31. M. Kojima, H. Hosoda, H. Matsuo, K. Kangawa. Ghrelin: discovery of the natural endogenous ligand for the growth hormone secretagogue receptor. Trends Endocrinol Metab, 12 (2001), pp. 118-122

32. M. Perez-Fontán, F. Cordido, A. Rodriguez-Carmona, R. Garcia- Naveiro, M.L. Isidro, P. Villaverde, et al. Acute plasma ghrelin and leptin responses to oral feeding or intraperitoneal hypertonic glucose-based dialysate in patients with chronic renal failure. Kidney Int, 68 (2005), pp. 2877-2885

33. K.G. Murphy, W.S. Dhillo, S.R. Bloom. Gut peptides in the regulation of food intake and energy homeostasis. Endocr Rev, 27 (2006), pp. 719-727

34. J.-P. Chanoine. Individual differences in the hormonal control of appetite: A step toward a (more) successful treatment of childhood overweight?. J Clin Endocrinol Metab, 91 (2006), pp. 2864-2866

35. A.P. Coll, S. Farooqi, S. O'Rahilly. The hormonal control of food intake. Cell, 129 (2007), pp. 251-262

36. D.E. Cummings, J. Overduin. Gastrointestinal regulation of food intake. J Clin Invest, 117 (2007), pp. 13-23

37. M. Broome, T. Hokfelt, L. Terenius. Peptide YY (PYY)-immunoreactivity neurons in the lower brain stem and the spinal cord of rat. Acta Physiol Scand, 125 (1985), pp. 349-352

38. M.M. Hagan. Peptide YY: a key mediator of orexigenic behavior. Peptides, 23 (2002), pp. 377-382

39. Kanatani, S. Mashiko, N. Murai, N. Sugimoto, J. Ito, T. Fukuroda, et al. Role of the Y1 receptor in the regulation of neuropeptide Y-mediated feeding: comparison of wild-type, Y1 receptor-deficient, and Y5 receptor-deficient mice. Endocrinology, 141 (2000), pp. 1011-1016

40. V. Scott, N. Kimura, J.A. Stark, S.M. Luckman. Intravenous peptide YY3-36 and Y2 receptor antagonism in the rat: effects on feeding behavior. J Neuroendocrinol, 17 (2005), pp. 452-457

41. S. Koda, Y. Date, N. Murakami, T. Shimbara, T. Hanada, K. Toshinai, et al. The role of the vagal nerve in peripheral $\mathrm{PYY}_{3-36}$-induced feeding reduction in rats. Endocrinology, 146 (2005), pp. 2369-2375

42. T.H. Moran, U. Smedh, K.P. Kinzig, K.A. Scott, S. Knipp, E.E. Ladenheim. Peptide YY(3-36) inhibits gastric emptying and produces acute reductions in food intake in rhesus monkeys. Am J Physiol Regul Integr Comp Physiol, 288 (2005), pp. R384-R388

43. Gantz, N. Erondu, M. Mallick, B. Musser, R. Krishna, W.K. Tanaka, et al. Efficacy and safety of intranasal peptide YY3-36 for weight reduction in obese adults. J Clin Endocrinol Metab, 92 (2007), pp. 1754-1757

44. M. Imamura. Effects of surgical manipulation of the intestine on peptide YY and its physiology. Peptides, 23 (2002), pp. 403-407

45. K. Zwirska-Korczala, S.J. Konturek, M. Sodowski, M. Wylezol, D. Kuka, P. Sowa, et al. Basal and postprandial plasma levels of PYY, ghrelin, cholecystokinin, gastrin and insulin in women with moderate and morbid obesity and metabolic syndrome. J Physiol Pharmacol, 58 (Suppl 1) (2007), pp. 13-35 
46. K. Højlund, M. Wildner-Christensen, O. Eshøj, C. Skjærbæk, J.J. Holst, O. Koldkjær, et al. Reference intervals for glucose, $\beta$-cell polypeptides, and counterregulatory factors during prolonged fasting. Am J Physiol Endocrinol Metab, 280 (2001), pp. E50-E58

47. M. Arosio, C.L. Ronchi, P. Beck-Peccoz, C. Gebbia, C. Giavoli, V. Cappiello, et al. Effects of modified sham feeding on ghrelinlevels in healthy human subjects. J Clin Endocrinol Metab, 89 (2004), pp. 51015104

48. O. Ukkola. Ghrelin and insulin metabolism. Eur J Clin Invest, 33 (2003), pp. 183-185

49. Asakawa, A. Inui, T. Kaga, G. Katsuura, M. Fujimiya, M.A. Fujino, et al. Antagonism of ghrelin receptor reduces food intake and body weight gain in mice. Gut, 52 (2003), pp. 947-952

50. T. McLaughlin, F. Abbasi, C. Lamendola, R.S. Frayo, D. Cummings. Plasma ghrelin concentrations are decreased in insulin-resistant obese adults relative to equally obese insulin-sensitive controls. J Clin Endocrinol Metab, 89 (2004), pp. 1630-1635

51. D.E. Cummings, J.Q. Purnell, R.S. Frayo, K. Schmidova, B.E. Wisse, D.S. Weigle. A preprandial rise in plasma ghrelin suggests a role in meal initiation in humans. Diabetes, 50 (2001), pp. 1714-1719

52. M. Tschop, C. Weyer, P.A. Tataranni, V. Devanarayan, E. Ravussin, M.L. Heiman. Circulating ghrelin are decreased in human obesity. Diabetes, 50 (2001), pp. 707-709

53. D.L. Williams, D.E. Cummings, H.J. Grill, J.M. Kaplan. Meal-related ghrelin suppression requires postgastric feedback. Endocrinology, 144 (2003), pp. 2765-2767

54. J. Nedvidkova, I. Krykorkova, V. Bartak, H. Papezova, P.W. Gold, S. Alesci, et al. Loss of meal-induced decrease in plasma ghrelin levels in patients with anorexia nervosa. J Clin Endocrinol Metab, 88 (2003), pp. 1678-1682

55. P. Lucidi, G. Murdolo, C. Di Loreto, N. Parlani, A. De Cicco, A. Ranchelli, et al. Meal intake similarly reduces circulating concentrations of octanoyl and total ghrelin in humans. J Endocrinol Invest, 27 (2004), pp. RC12-RC15

56. M. Patterson, K.G. Murphy, C.W. Le Roux, M.A. Ghatei, S.R. Bloom. Characterization of ghrelin-like immunoreactivity in human plasma. J Clin Endocrinol Metab, 90 (2005), pp. 2205-2211

57. R. Al Awar, O.A. Obeid, N. Hwalla, S. Azar. Postprandial acylated ghrelin status following fat and protein manipulation in healthy young women. Clin Sci (Lond), 109 (2005), pp. 405-411

58. M. Perez-Fontán, A. Rodriguez-Carmona, F. Cordido, J. Garcia- Buela. Hyperleptinemia in uremic patients undergoing conservative management, peritoneal dialysis, and hemodialysis: A comparative analysis. Am J Kidney Dis, 34 (1999), pp. 824-831

59. M. Perez-Fontán, F. Cordido, A. Rodriguez-Carmona, J. Peteiro, R. Garcia-Naveiro, J. Garcia-Buela. Plasma ghrelin levels in patients undergoing haemodialysis and peritoneal dialysis. Nephrol Dial Transplant, 19 (2004), pp. 2095-2100

60. M.L. Isidro, F. Cordido. Growth hormone secretagogues. Comb Chem High T Scr, 9 (2006), pp. 175-180

61. P. Alvarez-Castro, M.L. Isidro, J. Garcia-Buela, C. Dieguez, F.F. Casanueva, F. Cordido. Effect of acute ghrelin administration on glycaemia and insulin levels in obese patients. Diabetes Obes Metab, 8 (2006), pp. 555-560

62. M.L. Isidro, M. Perez-Fontan, A. Rodriguez-Carmona, J. Garcia- Buela, F. Cordido. Postprandial plasma levels of acylated and total ghrelin after a mixed meal. Obes Metab, 2 (2006), pp. 60-67

63. O. Giménez-Palop, G. Giménez-Pérez, D. Mauricio, J.M. González- Clemente, N. Potau, E. Berlanga, et al. A lesser postprandial suppression of plasma ghrelin in Prader-Willi syndrome is associated with low fasting and a blunted postprandial PYY response. Clin Endocrinol, 66 (2007), pp. 198-204

64. J. Kim B-, O.D. Carlson, J. Hang H-, D. Elahi, C. Berri, J.M. Egan. Peptide YY is secreted after oral glucose administration in a gender-specific manner. J Clin Endocrinol Metab, 90 (2005), pp. 6665-6671 